\title{
Monte Carlo calculations of track structures 7
}

\section{Hans Bichsel. Nuclear Physics Laboratory, Lniversity of Washington. Seattle WA $98195-4290$}

\section{INTRODUCTION}

In the study of radiation effects in matter, a knowledge of the spatial distribution of secondary products and of energy deposited is important. For charged particies heavier than electrons, energy is deposited along an almost straight track which can be seen visually in photographic emulsions or cloud chambers. The initial effect of the energy deposition is the production of clouds of collectively excited electrons ("plasmons"), ions and secondary electrons (" $\delta$ rays" which in turn produce plasmons etc). We describe the spatial pattern of energy deposited and new species produced as the track structure. The discussion presented here is only concerned with the initial track structure, the subsequent fate of the products is discussed in other papers of this Conference.

From quantum mechanical considerations we assume the interaction of a charged particle to be a collision in which it loses a discrete amount of energy. A track is formed by successive collisions randomly distributed along the particle path. with a random energy loss associated with each collision. While energetic $\delta$ rays are produced infrequently, it will be necessary to include their effects in the pattern of the track structure, Fig. 1.

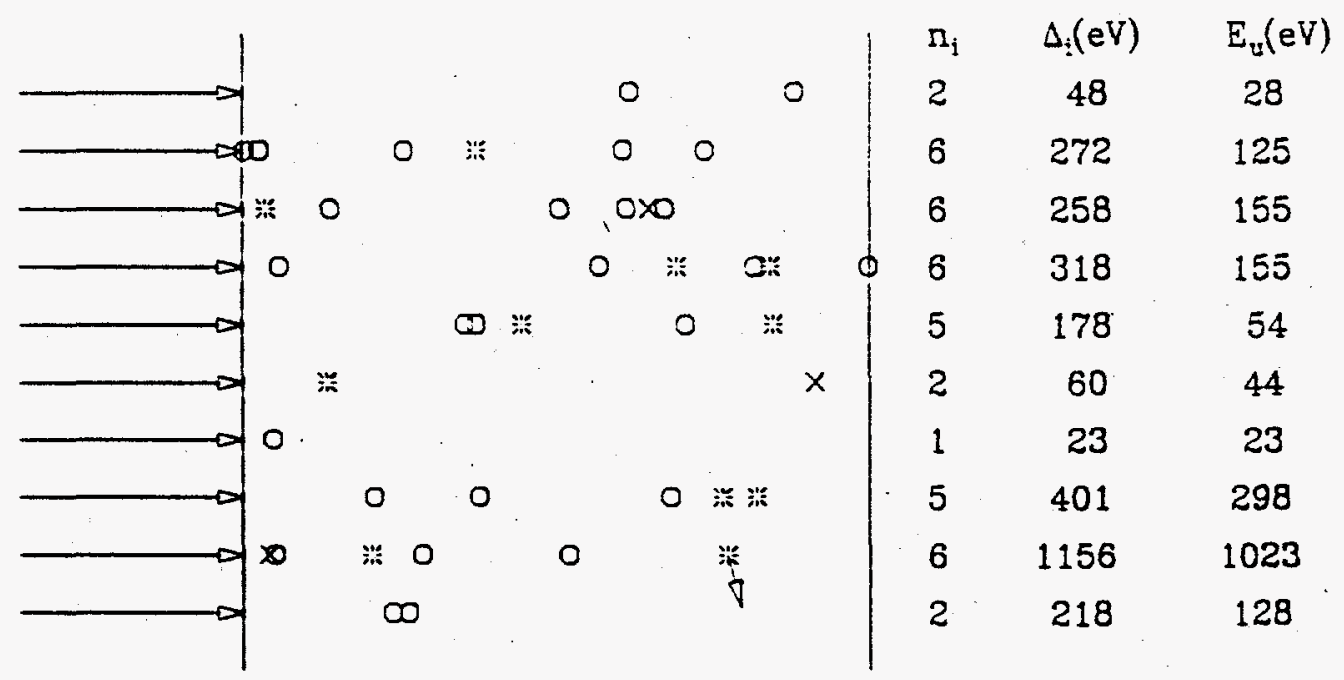

Fig. 1. Monte Carlo simulation of the energy loss of $100 \mathrm{MeV}$ protons in $t=424$ $\mathrm{nm}$ of liquid water. The velocity of the protons is represented by the arrow. The symbols $\mathrm{x}, \mathrm{o},{ }^{*}$ show the location of a collision and the associated energy loss $E_{i}$, see Fig. 2. With $\lambda=106 \mathrm{~nm}$, there are on the average $\langle n\rangle=t / \lambda=4$ collisions. The number of collisions $n_{i}$ for each proton, its total energy loss $\Delta_{i}=\sum E_{i}$ and the largest value $E_{u}$ are given. The average energy loss. calculated with the stopping power $S$ is $\langle\Delta\rangle=t \cdot S=307 \mathrm{eV}$. The practical range $R_{p}$ of the $E_{u}=1023 \mathrm{eV} \delta$ ray is shown by an arrow. For all other $\delta$ rays. $R_{p}$ is less than the size of the symbols. 


\section{DISCLAIMER}

This report was prepared as an account of work sponsored by an agency of the United States Government. Neither the United States Government nor any agency thereof, nor any of their employees, makes any wartanty, express or implied, or assumes any legal liability or responsibility for the accuracy, completeness, or usefulness of any information, apparatus, product, or process disclosed, or represents that its use would not infringe privately owned rights. Reference herein to any specific commercial product, process, or service by trade name, trademark, manufacturer, or otherwise does not necessarily constitute or imply its endorsement, recommendation, or favoring by the United States Government or any agency thereof. The views and opinions of authors expressed herein do not necessarily state or reflect those of the United States Government or any agency thereof. 


\section{DISCLAIMER}

Portions of this document may be illegible in electronic image products. Images are produced from the best available original document. 
The knowledge of track structure is important in all applications in which radiation effects are observed: In high energy physics, the spatial distribution and momentum of particles produced in nuclear collisions must be measured precisely. In a time projection chamber ("TPC") ionization is measured electronically in a way equivalent to the visual observation in a cloud chamber. A track segment in which an energetic delta ray is produced is not useful for the determination of particle momentum, and the track structure determines the ultimate limit of the precision with which the momentum can be measured. Similarly, in the detection of Röntgen rays with a segmented silicon detector, the energy deposition in the "pixels" is determined by track structure. In biological materials, track structure is an important element in the determination of radiobiological effectiveness ("RBE") and of effects on DNA. In the Fricke dosimeter and in plastic scintillators, the effect observed is related to track structure.

An extensive discussion of track structure for photons, neutrons, electrons and heavier particles is given in a recent IAEA report [1]. It includes an extensive discussion of chemical and biological effects and a list of 260 references.

The measurement of track structures in condensed matter at microscopic levels is difficult. Thus we use calculations to simulate them. On the other hand it is possible to make measurements in gases for dimensions equivalent to $\mu \mathrm{m}$ and even $\mathrm{nm}$ in the condensed phase.

\section{CALCULATION OF TRACK STRUCTURE}

In order to simulate the random nature of the particle interactions, Monte Carlo calculations must be made. Two primary quantities are needed for such a calculation:

a) the macroscopic total collision cross section $\Sigma_{t}=n \sigma_{t}$ where $n$ is the number of electrons per unit volume, or its inverse, $\lambda$, the mean free path length between collisions is used to determine the locus of an energy loss event. For particles with speed $\beta=v c$ and charge $z e$ we have

$$
\sigma_{t}=\frac{k z^{2}}{\beta^{2}} \tau
$$

with $k=2 \pi e^{4} / m c^{2}=2.55 \cdot 10^{-19} \mathrm{eV} \mathrm{cm}^{2}$ and $\tau=A\left[\log \left(\beta^{2} \gamma^{2}\right)-\beta^{2}\right]+B$ where $\gamma^{2}=1 /\left(1-\beta^{2}\right)$ and $A$ and $B$ are constants. For liquid water $A \sim 0.23, B \sim 2.4$, and $n=3.3 \cdot 10^{23} \mathrm{~cm}^{-3}$.

b) the cross section $\sigma(E, \theta)$ differential in energy loss $E$ and in angular distribution $\theta$ of the $\delta$ rays is used to determine the energy loss in the collision and the initial direction of the outgoing electron. Usually, the angular and the loss dependence of $\sigma(E, \theta)$ are separated. The cross section $\sigma(E)$ differential in $E$ can be approximated by the Fermi-virtual-photon method (FVP) as

$$
\sigma(E)=\rho(E)\left[E f(E) \log \frac{2 m v^{2}}{E}+\int_{0}^{E} f\left(E^{\prime}\right) d E^{\prime}\right]
$$

where $\rho(E)=k z^{2} /(\beta E)^{2}$ is the Rutherford cross section, and $f(E)$ is the dipole oscillator strength (DOS) for the optical absorption of photons with energy $E$. It must be noted that there is a considerable difference in $f(E)$ for liquid water and its vapor [2]. In the Monte 
Carlo calculations. for the determination of the energy loss. the quantity $Q(E)$ is used

$$
Q(E)=\frac{\int_{0}^{E} \sigma\left(E^{\prime}\right) d E^{\prime}}{\sigma_{t}} \text {. with } \sigma_{t}=\int_{0}^{\infty} \sigma\left(E^{\prime}\right) d E^{\prime} .
$$

it is shown in Fig. 2. It is remarkable how little $Q(E)$ depends on 3. Note especially that more than $7.5 \%$ of all collisions occur with energy losses of less than $40 \mathrm{eV}$, and only about $3 \%$ produce an energy loss exceeding $200 \mathrm{eV}$.

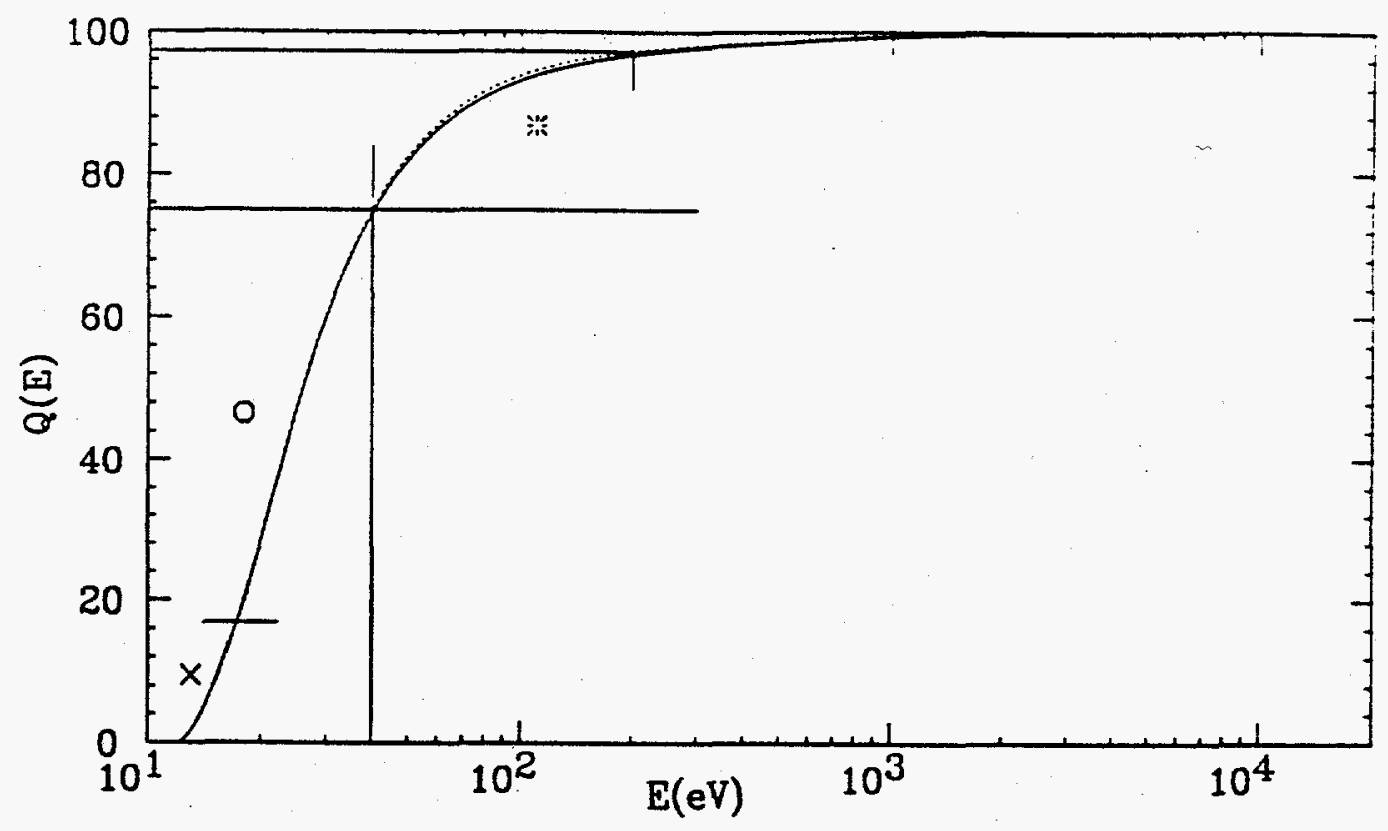

Fig. 2. Distribution function $Q(E)$ for energy losses less than $E$ in single collisions in water for $6 \mathrm{MeV}(\beta=0.11$, dotted line) and $100 \mathrm{MeV}(\beta=0.43$, continuous line) protons. The energy intervals for the symbols used in Fig. 1 are also given. The most probable energy. loss is at $\sim 22 \mathrm{eV}$.

The Monte Carlo calculation consists of four steps

a) a random number $r$ with values between 0 and 1 is used to determine the distance between successive collisions

$$
x_{i}=-\lambda \log r,
$$

b) the energy loss $E$ is selected with a second random number by entering it on the ordinate of Fig. 2 and reading the value of $E$ from the abscissa,

c) transport of $\delta$ rays,

d) a recording or classification of the pattern for each collision.

The average time between collisions is $t_{c}=\lambda / v$, of the order of $10^{-15} \mathrm{sec}$. In water, the initial species produced are $\mathrm{H}_{2} \mathrm{O}^{+}$and other ions, plasmons, excited molecules and subex- 
citation electrons, and the physical processes take place in less than $10^{-12} \mathrm{sec}$. The processes taking place during the first $\mu \mathrm{sec}$ after the collisions are considered to be chemical in nature, leading to a variety of radicals diffusing away from the track. Biological processes follow this stage and may last for hours [3].

\section{COMPARISON WITH EXPERIMENTS}

The accuracy of Monte Carlo calculations is determined by the accuracy of the differential collision collision spectrum. The approximation given by Eq. (2) can be improved by the Bethe theory based on experimental scattering experiments [4]. So far, experiments with very few collisions in condensed matter have only been made for electrons. We are confident that the extrapolation from electrons to heavier particles is reliable, but it is important to compare Monte Carlo results for heavy particles with experimental data. This is quite easy for gases. E.g. in microdosimetry, the ionization produced by each passing ion through a defined volume is recorded. This process can readily be simulated by summing the calculated collision patterns over the defined volume of the instrument [5]. A different geometry is used in TPCs, but can readily be simulated, too. For condensed matter, observations in small volumes can be made e.g. in silicon devices (CCDs), bubble chambers and photographic emulsions. It is also possible to observe track structure effects in macroscopic volumes such as in thermoluminescent dosimeters, Fricke solutions, track etching etc.

Because the chemical species (ions, radicals etc.) produced by the radiation will interact with DNA and other organic molecules in biological materials, in addition to the initial direct direct excitations and dissociations, it is difficult to relate track structure to biological effects [3]. Furthermore, an RBE will depend on cell cycle, nutrients etc.

\section{CONCLUSIONS}

It is important to balance the complexity of a Monte Carlo code against the computation time and uncertainty permissible for the results. For applications to biological structures it may be necessary to make a separate Monte Carlo calculation for each collision of each $\delta$ ray. For the simulation of microdosimetric spectra, it may be sufficient to consider an energy deposition function derived from experimental data [6]. In some applications it will be important to follow the fate of electrons to energies below the lowest possible excitation in the absorber ("sub-excitation electrons" [1]).

For cancer therapy with charged particle beams protons with energies between 60 and $250 \mathrm{MeV}$ and heavy ions (carbon, neon) with energies of several hundred $\mathrm{MeV}$ per nucleon are used. A major advantage of these beams is their production of a Bragg peak, i.e. a small region of high dose near the end of their range. For heavy ions there is a slight disadvantage due to the presence of a small dose beyond the extrapolated range, caused by fragments (protons, $\alpha$ particles etc) produced by nuclear collisions of the beam particles. In order to establish a good treatment regime, it is important to know the RBE of the beam as a function of its depth of penetration $t$ in the irradiated tissues. This can be related to 
the track structure. In order to assess the effort which should be made to establish the track structure as a function of residual particle energy, it is useful to consider the energy spectrum of the beam as a function of depth $t$. A clear distinction must be made between the ionization density $J_{1}$ along the path of a single ion. and that of the whole beam. $J$. For a broad 290 $\mathrm{MeV} / \mathrm{u}$ beam of carbon ions. the ratio of the ionization at the maximum of th: Bragg peak to that at $t=0$ is about $5: 1$. for a single ion, it is about $40: 1$. For a single particie. this ratio is very large over only a very short range of energies ( $\mathrm{a}$ few $\mathrm{MeV}$ ), while in a treatment beam with an extended region of high dose, most of the ions have a much higher energy at any given depth. The reason for this is that energy loss straggling mainly determines the shape of the Bragg curve. Thus. for the use in radiation therapy, it may not be necessary to obtain detailed results for the end of the range of each ion. If the treatment volume has a depth of $5 \mathrm{~cm}$ and is treated to a dose of $2 \mathrm{~Gy}$, cells on the average will be traversed by 36 ions, but in only one of about 140 cells an ion will stop, and the contribution from stopping ions is not very important, even though its stopping power is high.

For the simulation of micro-dosimetric measurements, calculation time can be greatly reduced if $\lambda$ is much smaller than the smallest dimension of the measurement volume: a "core track" for energy losses less than say $500 \mathrm{eV}$ can be calculated analytically [4], and a Monte Carlo calculation is only needed for greater energy losses, i.e. less than $1 \%$ of the collisions, Fig. 2.

\section{References}

[1] "Atomic and molecular data for radiotherapy and radiation research" IAEA-TECDOC799, published by the International Atomic Energy Agency May 1995

[2] M. Zaider, M. G. Vracko, A. Y. C. Fung and J. L. Fry, Radiat. Protec. Dosimetry 52, 139-146 (1994)

[3] Aloke Chatterjee and William R. Holley, Advances in Radiation Biology 17, 181-226 (1993)

[4] "Atomic, molecular and optical physics reference book", ed. G. W. F. Drake, to be published by the American Institute of Physics, 1995

[5] Hans Bichsel, Radiat. Protec. Dosimetry 13, 91-94 (1985)

[6] E. Waibel and B. Grosswendt, Nucl. Instr. Meth. 211, 487-498 (1983), and Phys. Med. Biol. 37, 249-259 (1992). 